\title{
New cosmological model including inflation, deceleration, acceleration and deceleration again
}

\section{P.V.N.M.C. Perera ${ }^{1}$, K.D.W.J. Katugampala ${ }^{2}$}

\begin{abstract}
Since Perlmutter and A.G. Riess (Reiss, Adam G.,1998) observed that the Universe expands with an acceleration, many models involving dark energy have been proposed to explain this phenomenon. (Perlmutter, S., 1997), (Reiss, Adam G.,1998). A family of cosmological models with both acceleration and deceleration is presented in this research study. According to Einstein's field equations, in general, relativity is in the form,

$$
R^{\mu \nu}-\frac{1}{2} \bar{R} g^{\mu \nu}=k T^{\mu \nu}-\Lambda g^{\mu \nu}
$$

The Einstein's field equations are modified in this research study. We consider $\Lambda$ a parameter of cosmic time. The assumptions of a homogeneous and isotropic universe are based on the Mach's principle. We started with the Robertson-Walker metric in spherical polar coordinates. We found the Christoffel symbols to define the Ricci tensor, the curvature scalar and the energy-momentum tensor using the Robertson-Walker metric. The modified Einstein's field equations for scalar factor $R(t)$ which is called "radius of the universe" is solved using the
\end{abstract}

1 Department of Mathematics, Faculty of Engineering, The General Sir John Kotelawala Defence University, Ratmalana, Sri Lanka, niroshmcperera@gmail.com

2 Department of Mathematics, University of Kelaniya, Sri Lanka, wasantha@kln.ac.lk

Date Received: $\quad 02^{\text {nd }}$ November 2018

Date Accepted: $\quad 14^{\text {th }}$ November 2019 
Robertson-Walker metric and Energy momentum tensor.The solution of $R(t)$ can be introduced in the following form, so that it shows the inflation at the beginning.

$$
R(t)=b \sqrt{\left(1-\cos ^{3} \omega t\right)}
$$

A solution is assumed for the universe which results in inflation, deceleration, acceleration and deceleration again. Taking the present value of the cosmic time $t$ as 13.7 billion years we find the density of the inflationary universe is $2.0211 \times$ $10^{-28} \mathrm{Kgm}^{-3}$ and deceleration of the universe is $9.1822 \times 10^{3} \mathrm{~ms}^{-2}$ which agree with the observations. We discussed the redshift of light from extragalactic sources, which arises from the Robertson-Walker metric. This redshift is a measure of the expansion of the universe in a given period of time. The redshift is the ratio of the value of the scalar factor of the universe at present epoch to that at the epoch of emission of light from the extragalactic sources which is observed at present. The scalar factor is increasing with time $t$ at present. However, there could be epochs where the scalar factor is decreasing.

Keywords: cosmological model, scalar factor, Einstein's field equation, inflation.

\section{Introduction}

In 1997 Perlmutter and his group, and in 1998 Reiss and his group from the observations of Type 1A supernovae concluded that the universe is not only expanding but expanding with an acceleration. (Perlmutter, S., 1997), (Reiss, Adam G.,1998). This came as a surprise as the standard model had been well established for some time which explained the big bang microwave background radiation and other important observations. According to the standard model the universe has been expanding with a deceleration after an initial inflation as postulated by Alan Guth though without a proper mechanism (Reiss, Adam G., 1998).

Various theories have been proposed to explain the expansion of the universe with acceleration and it has been generally believed that this acceleration is due to what is called, "dark energy". However, the problem has been to identify the dark energy. The vacuum energy had been one of the candidates and there were many 
who identified vacuum energy with the cosmological constant. (Weinberg S., 1989) However, there was a problem as the cosmological term was very small and the vacuum energy was very large, and the difference being of order 150 . However, there were others who had referred to the cosmological constant and vacuum energy separately while some invoked quintessence to explain the so called dark energy. (Sato, K. ,1981)

\section{Methodology}

In order to explain dark energy, we modified Einstein's field equations and considered the cosmological constant giving rise to an energy field and included the cosmological term on the right hand side of the field equations instead of on the left hand side. The terms on the left hand side of the equations referred to the geometry while the terms on the right hand side represent matter radiation and energy. Thus the cosmological term was transferred from being a term representing geometry of the space time to that representing energy and it is also considered a parameter of cosmic time, and not a constant as was done originally by Einstein.

$$
R^{\mu \nu}-\frac{1}{2} \bar{R} g^{\mu \nu}=\kappa T^{\mu \nu}-\Lambda g^{\mu \nu}
$$

Next we consider the Robertson-Walker metric,

$$
d s^{2}=c^{2} d t^{2}-R^{2}(t)\left[\frac{d u^{2}+u^{2}\left(d \theta^{2}+\sin ^{2} \theta d \phi^{2}\right)}{\left(1+\frac{\kappa u^{2}}{4}\right)^{2}}\right]
$$

and evaluate non-vanishing Christoffel symbols of Robertson-Walker metric.

The $\Lambda$ term introduced by Einstein himself gives rise to a field that repels particles and objects rather than to one that attracts them. For the different values of $\mu$ and $v$ in equation (1), we obtained following two independent equations with four unknown variables $R, \rho, \Lambda$ and $p$. 


$$
\begin{aligned}
& \kappa \rho=\Lambda-\frac{3 \dot{R}^{2}}{R^{2} c^{2}}-\frac{3 \kappa}{R^{2}} \\
& \frac{\kappa p}{c^{2}}=-\Lambda+\left[\frac{\kappa}{R^{2}}+\frac{\dot{R}^{2}+2 R \ddot{R}}{R^{2} c^{2}}\right]
\end{aligned}
$$

In order to use the Robertson-Walker metric we should decide which value of the three possible values $\kappa=-1,0,+1$ would give an appropriate solution. We write the equations (3) and (4) as

$$
\frac{8 \pi G}{c^{2}}\left(\rho+\frac{p}{c^{2}}\right)=2\left(\frac{\kappa c^{2}+\dot{R}^{2}-R \ddot{R}}{R^{2} c^{2}}\right)
$$

Physical quantities pressure and density should remain positive and this implies that the left hand side of (5) should be positive. Then the only possible value of $\kappa$ is +1

The universe is believed to have expanded with an inflation. To support this inflation we selected the following form of equation, therefore the inflation could be accommodated.

We assume that a family of solutions of above equations for $R$ can be written in the form,

$$
R(t)=b \sqrt{\left(1-\cos ^{3} \omega \mathrm{t}\right)}
$$

using the following boundary conditions,

- $R=0$ at $t=0$,

- $\dot{R}=0, \ddot{R}=0$ at $\omega t=\frac{\pi}{2}$, (point of inflection),

- The present value of rate of expansion of universe is $7.43 \times 10^{4} \mathrm{~ms}^{-1}$ where $b$ and $\omega$ are unknown constants.

Recent observations have led to the approximate value $\frac{7}{3}$ for the ratio of dark energy $\left(\Lambda^{\prime}=\frac{\Lambda c^{2}}{8 \pi G}\right)$ to matter density $(\rho),\left[\frac{\Lambda^{\prime}}{\rho}\right]$ and to the value 1.6 , for the 
redshift $\left[\frac{\left.R\right|_{\omega t=\omega t_{0}}}{\left.R\right|_{\omega t=\frac{\pi}{2}}}\right]$, at the onset of acceleration. Taking this redshift to be a constant, a family of solutions can be found for different ratios of dark energy to matter density. Similarly, keeping the ratio of dark energy to matter density as $\frac{7}{3}$ we found that a family of solutions can be obtained for different values for the above redshift. Though there is no solution when the redshift is 1.6 , there is a solution when its value is 1.3 , which is good enough considering the uncertainties associated with measurements.

\section{Results and discussion}

The age of the universe is estimated to be 13.7 billion years. Taking the present value of the cosmic time $t$ as 13.7 billion years we find, $b=4.4168 \times 10^{20} \mathrm{~m}$, $\omega=6.8 \times 10^{-18} \mathrm{rad} \mathrm{s}^{-1}$ when the above redshift is 1.3 . The variation of $R(t)$ with $t$ is given in Figure(1). It is seen that, the "radius of the universe", $R(t)$ increases in the first phase with both acceleration and deceleration.

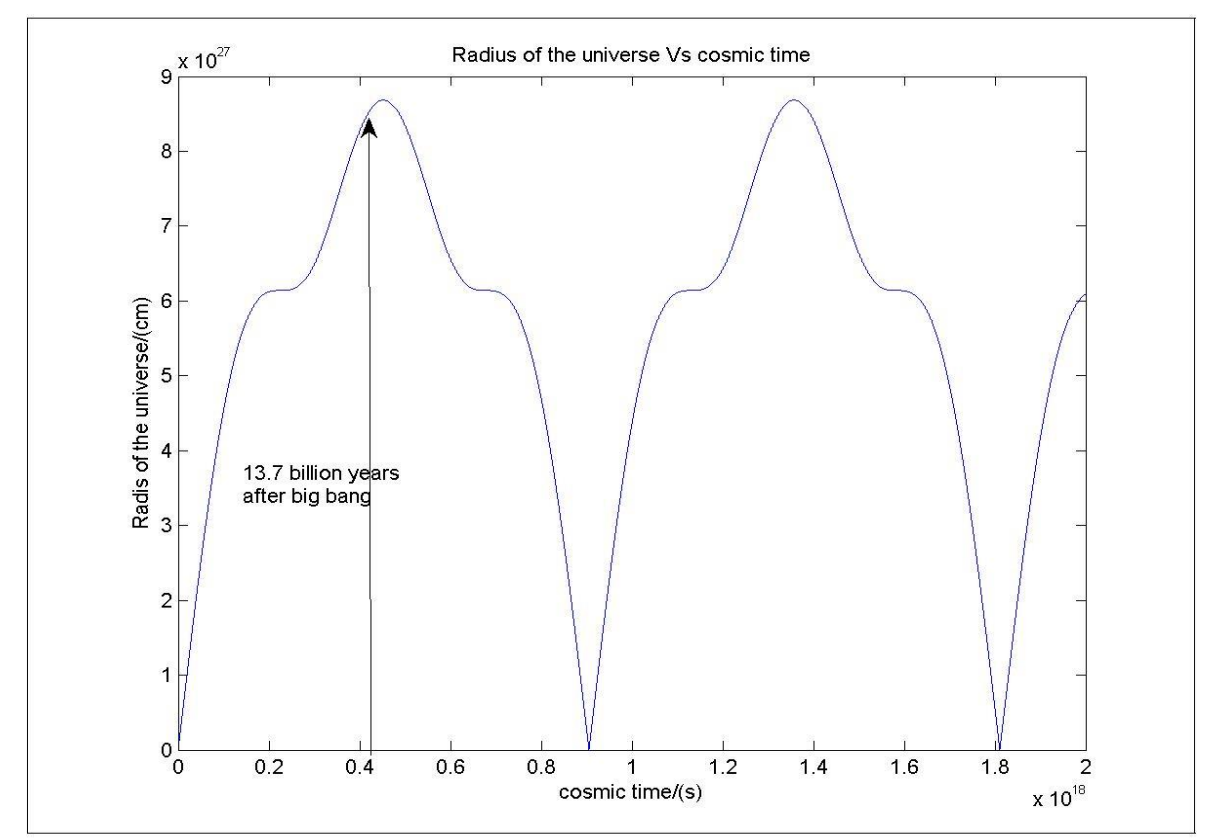

Figure 1. Radius of the Universe with respect to the solution $R(t)=$

A

$$
b \sqrt{\left(1-\cos ^{3} \omega t\right)}
$$

62 
The Figure 1 shows that the universe expands and contracts in cycles after the big bang. It is now in its expansion phase of the first cycle. The universe expands during phase $\mathrm{AB}$ with an acceleration then in $\mathrm{BC}$ with deceleration. At $\mathrm{C}$ it undergoes a point of inflection and starts acceleration again in phase $\mathrm{CD}$ following a deceleration in phase DE. After $E$ the universe starts contraction. The acceleration observed by Perlmutter could correspond to the phase $\mathrm{CD}$ and the deceleration claimed by Schaefer could be represented by the phase DE.

Taking the present value of the cosmic time $t$ as 13.7 billion years, we find the density of the inflationary universe is $2.0211 \times 10^{-28} \mathrm{Kgm}^{-3}$ and deceleration of the universe is $9.1822 \times 10^{3} \mathrm{~ms}^{-2}$ which agree with recent observations (Weinberg S., 1989).

\section{Conclusion}

In this research we postulated that the cosmological term arises out of the space time energy and the term is a measure of the space time energy. Writing down of the cosmological term on the right hand side of the field equations made the tensor $k T^{\mu \nu}-\Lambda g^{\mu \nu}$ divergenceless, and hence what is conserved is not the energy due to matter and radiation, but energy due to matter, radiation and space - time.

This is a new result in itself as it makes not only space time a form of energy but a form of energy that could be converted to other forms of energy and vice versa. Until Einstein formulated his Theory of general relativity space - time was the arena in which matter and radiation moved without changing the space - time itself. Einstein revolutionized this concept and said that matter and radiation changed the space - time as well. In other words according to Einstein, space time is determined by matter and radiation. Einstein wanted to incorporate Mach's Principle that implied that there was no space - time in the absence of matter or radiation. However, Einstein did not succeed in his attempt to incorporate Mach's Principle into his general theory of relativity. 
In this work we have gone a step further by taking space - time as a form of energy that could be converted into other forms of energy and vice versa, thus not only that matter and energy determine space - time but could get converted into space time. In a sense, Mach's Principle becomes superfluous as when space -time exists it implies that matter or radiation exists.

With the modification of the field equations and interpretation of space time as a form of energy we attempted to find solutions of the modified field equations. We imposed certain boundary conditions and our strategy was to assume a family of equations with certain arbitrary constants and find those constants using the boundary conditions. The field equations reduced to two independent equations and we had to solve them for four variables, the scalar factor $R$, the density $\rho$, the pressure $p$ and the cosmological Parameter $\Lambda$.

We found a family of solutions that satisfied the boundary conditions imposed and agreed with many of the observations by the cosmologists. The model accounted for the inflation at the beginning and the model predicted a universe that started with initial zero velocity but with an acceleration.

In this work, we attempted to propose a model for the universe which contained an expansion with both acceleration and deceleration. We have been able to show that our model agrees with the recent observations.

\section{References}

Blake, C., Brough, S., Colless, M., Contreras, C., Couch, W., Croom, S., Croton, D., (2012). The WiggleZ Dark Energy Survey: joint measurements of the expansion and growth history atz $<1$. Monthly Notices of the Royal Astronomical Society, 425(1), pp.405-414.,Viewed 21 June 2018, https://academic.oup.com/mnras/article/425/1/405/999843

Blake, C., Glazebrook, K., Davis, T.M., Brough, S., Colless, M., (2011). The WiggleZ Dark Energy Survey: measuring the cosmic expansion history using the Alcock-Paczynski test and distant supernovae. Monthly Notices of the Royal Astronomical Society, 418(3), pp.1725-1735. 
Jayant Narlikar (2002). An introduction to cosmology. Cambridge: Cambridge University Press.

McVittie, G.C. and Lindsay, R.B. (1957). General Relativity and Cosmology: Vol. 4 of the International Astrophysics Series. Physics Today, 10(5), pp.38-40.

Nasa.gov. (2012). Biography of Edwin Powell Hubble (1889 - 1953), Viewed 21 June 2018, https://asd.gsfc.nasa.gov/archive/hubble/ overview/hubble_bio.html

Nasa.gov. (2013). WMAP- Age of the Universe, viewed 16 March 2018, https://wmap.gsfc.nasa.gov/universe/uni_age.html

Perlmutter, S. (2003). Supernovae, Dark Energy, and the Accelerating Universe. Physics Today, 56(4), pp.53-60, Viewed 18 June 2018, https://physicstoday.scitation.org/doi/10.1063/1.1580050

Perlmutter, S., Gabi, S., Goldhaber, G., Goobar, A., (1997). Measurements of the Cosmological Parameters $\Omega$ and $\Lambda$ from the First Seven Supernovae atz $\geq 0.35$. The Astrophysical Journal, 483(2), pp.565-581.

Riess, A.G., Filippenko, A.V., Challis, P., (1998). Observational Evidence from Supernovae for an Accelerating Universe and a Cosmological Constant. The Astronomical Journal, 116(3), pp.1009-1038.

Sato, K. (1981). First-order phase transition of a vacuum and the expansion of the Universe. Monthly Notices of the Royal Astronomical Society, 195(3), pp.467479, viewed 15 May 2018, http://www.adsabs. harvard.edu/abs/ 1981MNRAS.195..467S

Weinberg, S. (1989). The cosmological constant problem. Reviews of Modern Physics, 61(1), pp.1-23. 
